إعداد خارطة الكترونية لتطوير حوض وادي الشور شمال شرق مدينة الموصل باستخدام

$$
\text { نظم المعلومات الجغرافية }
$$

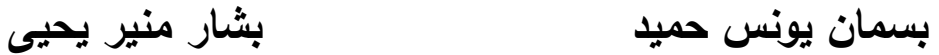

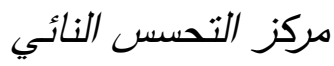

$$
\begin{aligned}
& \text { جامعة الدوصل }
\end{aligned}
$$

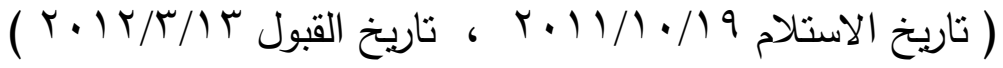

تتمثل منطقة الدراسة بحوض وادي الثنور والذي يقع شمال شرق مدينة الموصل،تضمنت الدراسة تطوير الحوض من الناحية الهيدرولوجية وأعداد خارطة الكترونية بالاعتماد على تقنيات التحسس النائي كأداة وفعالة في تحديد شبكة التصريف السطحية وتحليل الخواص المورفومترية لحوض وادي الثور ودراسة الموازنة العيدرولوجية وتحديد كميات المياه الداخلة والخارجة من الحوض مع تحديد الآبار الصالحة للثرب وللزراعة في المنطقة والعمل على تطوير إنتاجيتها عن طريق تصميم إحدى طرائق حصاد مياه الأمطار في منطقة الدراسة.تم جمع مستويات البيانات التي عولجت باستخدام برمجيات نظم المعلومات الجغرافية والنتائج

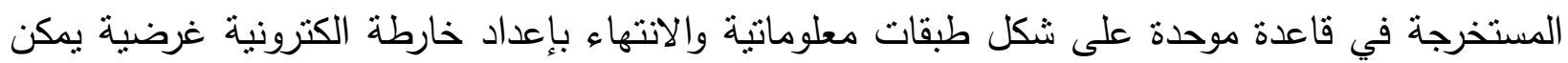
الاستفادة منها في مراقبة وتطوير منطقة الدراسة.

\title{
The Preparation of an Electronic Map for the Development of Wadi AL-shour Basin Valley North Eastern Mosul City Using Geographic Information System (GIS)
}

\author{
Basman Y. Hameed \\ Bashar M. Yahya
}

Remote sensing center

University of Mosul

\section{ABSTRACT}

The study area is within Wadi AL-Shour basin north eastern mosul city. This work includes the hydrological development of the basin and the preparation of an electronic map based on remote sensing technique. The technique is considered a 
very active tool for the determination of the surface drainage system, analysis of the morphomatric features of the basin and the study of the hydrological balance of the basin. It has also been used for the determination of input and output flow of basin waters, the suitability of the well waters for drinking and agricultural purposes and the improvement of the productivity of the basin through the design of a harvesting method for rain waters at the study area. The collected data is processed by the use of (GIS) software and the results obtained are gathered in a main data base as a digital layers, loading to the preparation of a purpose electronic map which could be used for the monitoring and developing the study area.

\section{المقدمة}

يقع حوض وادي الثور في الجزء الثمالي الثرقي من العراق وبالتحديد شرق مدينة الموصل وينحصر

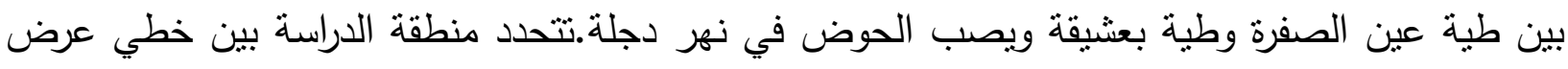

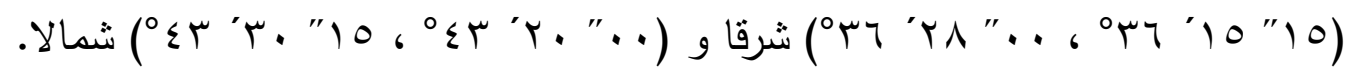

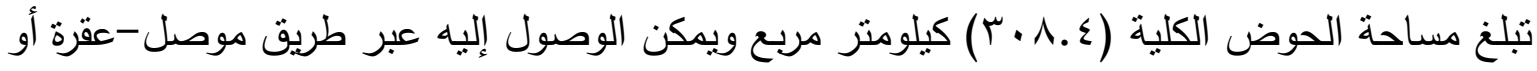

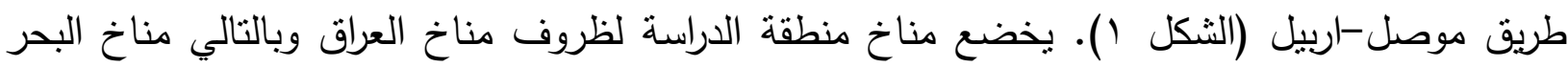

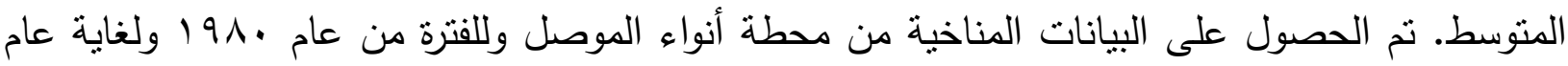

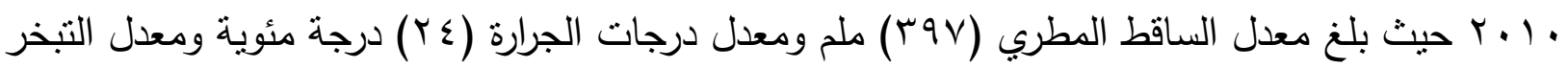
. $9 \cdot 9$ ) مل

إن مشكلة العجز المائي عززت أهمية التفكير الجدي بإدارة وصيانة وتطوير الأحواض النهرية عن

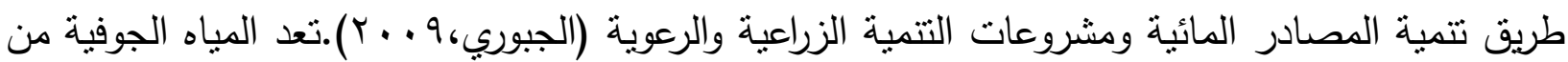
المصادر المهمة للمياه في الطبيعة وهي جزء من الدورة الهيدرولوجية لذا فان من الضروري معرفة كمياتها وكيفية العمل على زيادتها وذلك عن طريق دراسة الموازنة الهيدرولوجية للأحواض النهرية (ألنعيمي، $\cdot(r \cdot \cdots$

تم تصنيف المياه الجوفية في منطقة الدراسة حسب (جبرائيل، سلو I ( ) حيث تقع منطقة الدراسة ضمن القطاع (B2) والتكوين الجيولوجي هو تكوين الفارس وذات إنتاجية متغيرة ونوعية المياه توصف بأنها جيدة

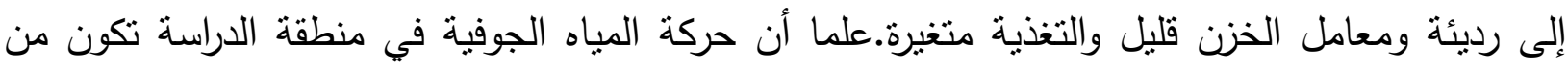

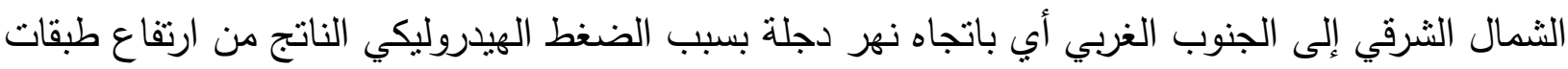
التكوين المائي عند أقدام الجبال في عين الصفرة وبعشيقة. تتصف التكوينات المائية الحاملة والخازنة للمياه الجوفية بأنها تكوينات مائية غير محصورة والمتنثلة بتكوين الانجانة والفتحة حيث أن المصدر الأساسي لتغذية هذين التكوينين هما مياه الساقط المطري السنوي الذي يسقط على المرتفعات المتمنلة بطية بعشيقة وعين الصفرة والتي تتسرب عبر الترسبات الحديثة ومنها 
تتفذ إلى تكويني الانجانة والفتحة حيث يعتبر تكوين الانجانة الخازن الرئيسي وبسمك ( •^) مثر، تم تحديد واحد وعشرين بئرا ارتوازيا في منطقة الدراسة وتم التركيز على تسعة منها وذلك لان مياهها تمتاز بنوعية جيدة صالحة للاستهلاك البشري حسب المواصفات العالمية والأمريكية (WHO, 1971). إن دراسة الخصائص المورفومترية المساحية والثكلية للأحواض النهرية يعطي دلالة واضحة على ده تحديد خواص الأحواض النهرية من ناحية كمية الموجات التصريفية وسرعتها مما يعطي مؤشرا دقيقا عن كيفية وضع الحلول الهندسية المناسبة للتعامل مع هذه الكميات المخمنة (المولى، ب . . ץ). يمكن تعريف مصطلح حصاد مياه الأمطار على انه فن قديم يقوم على مبدأ مسك وخزن الهاطل المطري بأثكال عدة مبتكرة حقليا تستتد على التغير الهائل في الاحتمالية المكانية والزمانية للهاطل المطري، يمكن استخدام مياه الأمطار المحصودة في مجالات شتى الزراعية منها وفي تربية المواشي وحتى للاستخدام البشري وحسب الطريقة المنفذة لحصاد هذه الأمطار ويمكن التعامل مع هذا الماء المحصود أما بتحويله مباشرة إلى أراضي زراعية لغرض الإرواء أو يتم خزنه بإحدى طرق الخزن المتمثلة بـ( قطاع التربة ، إنشاء

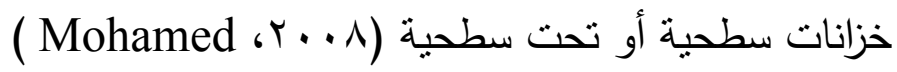
برزت أهمية تقنيات التحس النائي في الحصول على البيانات الضرورية التي تذعم تطوير الأحواض النهرية ومنها رسم شبكة التصريف السطحية لحوض وادي الثور بدقة عالية إضافة إلى استخدام برمجيات نظم المعلومات الجغرافية (GIS) في جدولة وتتظيم البيانات وإجراء التحاليل اللازمة للاراسة والانتهاء بإعداد خرائط غرضية تساعد في تطوير حوض وادي الثور (Kumar, 2008).

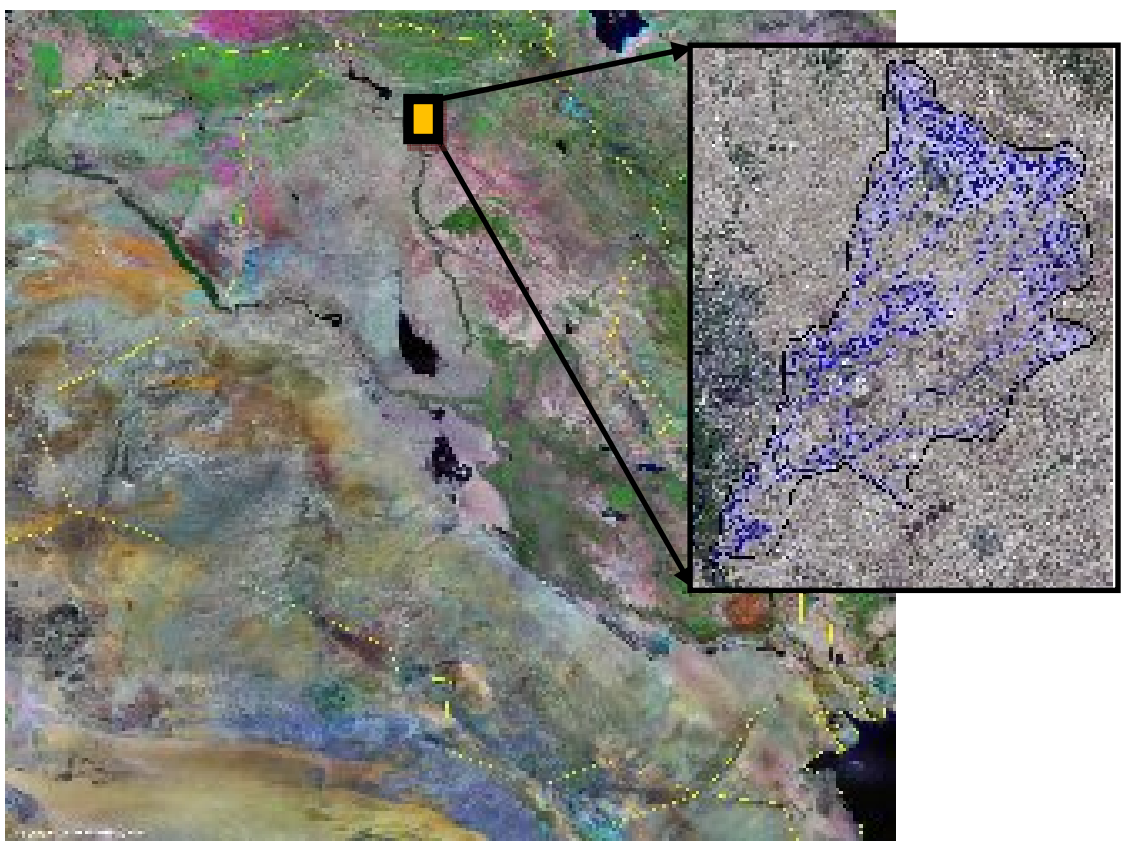

الثكل ا : مرئية فضائية تبين منطقة الدراسة. 


\section{بسمان يونس حميد و بشار منير يحيى}

\section{أهداف الدراسة وأهميتها}

1-دراسة الموازنة الهيدرولوجية لمنطقة الدراسة وتحديد كميات المياه الداخلة والخارجة من حوض وادي

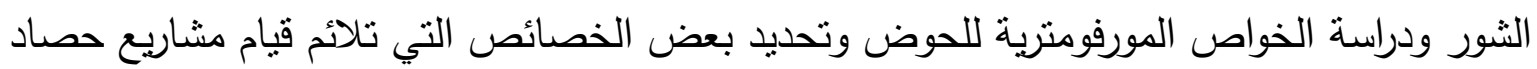
مياه الأمطار في منطقة الدراسة. ץ-استخدام تقنيات التحس النائي وبرمجيات نظم المعلومات الجغرافية في رسم شبكة التصريف السطحية والتحليل المورفومتري وإعداد قاعدة بيانات رقمية تحاكي البيانات المكانية والوصفية وإخراجها

$$
\text { على شكل خارطة الكترونية غرضية. }
$$

أن أهمية الدراسة تكمن في إعداد خارطة الكترونية تستخدم في مراقبة وتطوير الحوض من الناحية الهيدرولوجية وذلك بدراسة الموازنة الهيدرولوجية للمنطقة والخواص المورفومترية وتحديد كميات المياه الجوفية

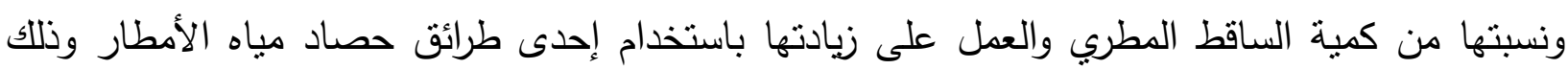
لتغذية الآبار الموجودة ضمن حوض الثور والصالحة للشرب وللزراعة ومن ثم جمع البيانات الداخلة والخارجة وتوحيدها في قاعدة بيانات طبقية يمكن عرضها بشكل خارطة الكترونية وتطويرها سنوية بإضافة

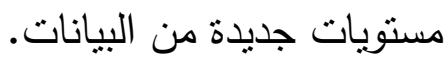

\section{البيانات والبرمجيات المستخدمة في الاراسة}

تم الاعتماد على البيانات التالية أثناء تتفيذ مراحل الدراسة وهي كالأتي: 1-البيانات المناخية لمنطقة الدراسة والتي نت الحصول عليها من محطة أنواء الموصل وللفترة من عام

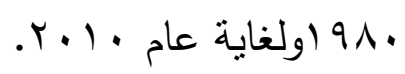

ץ-خارطة التصريف السطحية: تم الاستعانة بخارطة تصريف سطحية لحوض الوادي والمعدة مسبقا من

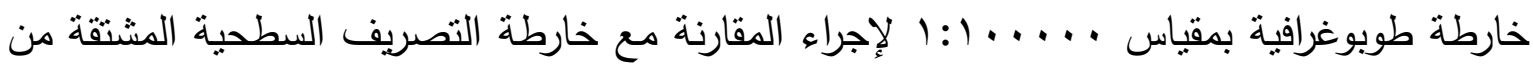

خلال استخدام برنامج (Arc map v.9).

ب-البيانات الفضائية: نم الاعتماد على البيان الفضائي والملتقط من قبل القمر الصنعي الأمريكي والذي يحمل على متته المتحسس من نوع راسم الخرائط الموضوعي الدحسن (Land sat)

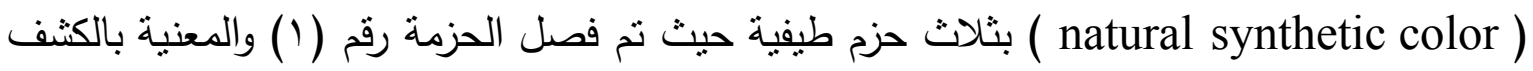

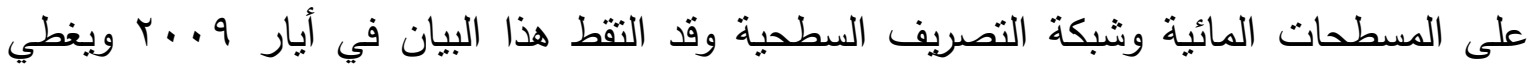

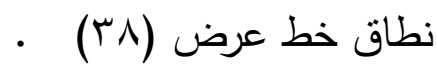


צ-نموذج الارتفاع الرقمي (DEM): تم الاعتماد على نموذج الارتفاع الرقمي( Digital Elevation) (Modal الكفاف بأي فترة كنتورية كما يستفاد منه في رسم المقاطع العرضية العاع العكانية وتكوين الأشكال المجسمة ثناثية

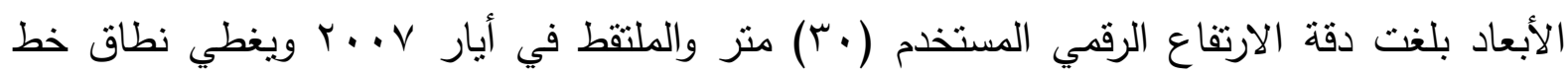

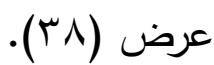

ه- البرامج الجاهزة المستخدمة:تستخدم في مجال أنظمة المعلومات الجغرافية (Geographical) information system software )(GISS)

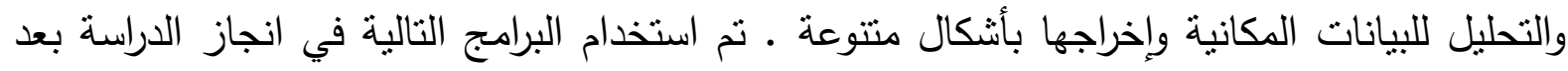
توفر البيانات اللازمة لتشغيل هذه البرامج الجدول (1).

الجدول ا: برمجيات نظم المعلومات الجغرافية والغاية من استخدامها في الدراسة.

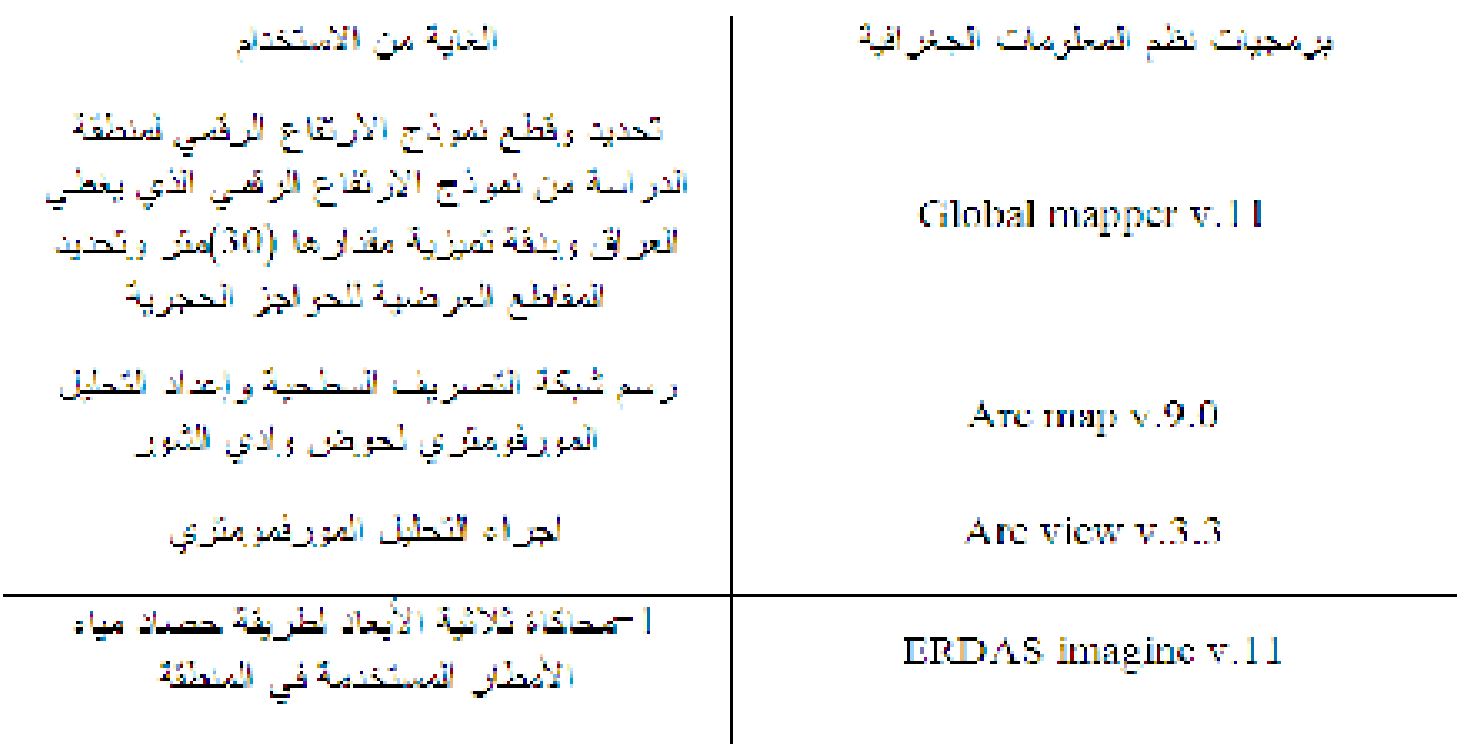




\section{منهجية إجراء الدراسة}

أولا: رسم شبكة التصريف السطحية

تم استخدام برنامج (Arc map v.9.0) في رسم شبكة التصريف السطحية لحوض وادي الشور بالاعتماد على نموذج الارتفاع الرقمي(DEM) لمنطقة الدراسة.تم مقارنة شبكة التصريف السطحية المنتجة مع الخارطة الطوبوغرافية لمنطقة الدراسة حيث كانت نتيجة التطابق ما بين التفسير البصري والخارطة الطوبوغرافية جيدة جدا (الثكل ب ب).

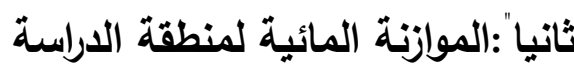

يمكن تعريف الموازنة الهيدرولوجية على أنها معادلة حساب النوازن بين كميات المياه الداخلة وكميات المياه الخارجة في أي حوض مائي وخلال فترة زمنية طويلة نسبيا مع توفر المكونات الأساسية لهذه المعادلة وهي الكميات الداخلة بواسطة الهاطل المطري والكميات الخارجة وهي الخزين الجوفي والمفقودة بالتبخر

$\mathrm{P}=\mathrm{R}+\mathrm{ET}+\mathrm{U}+\mathrm{S}_{\mathrm{s}}+\mathrm{S}_{\mathrm{g}}$ معادلة رقم

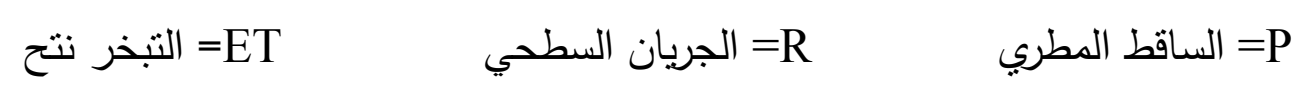

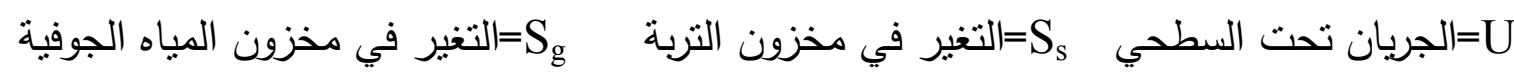

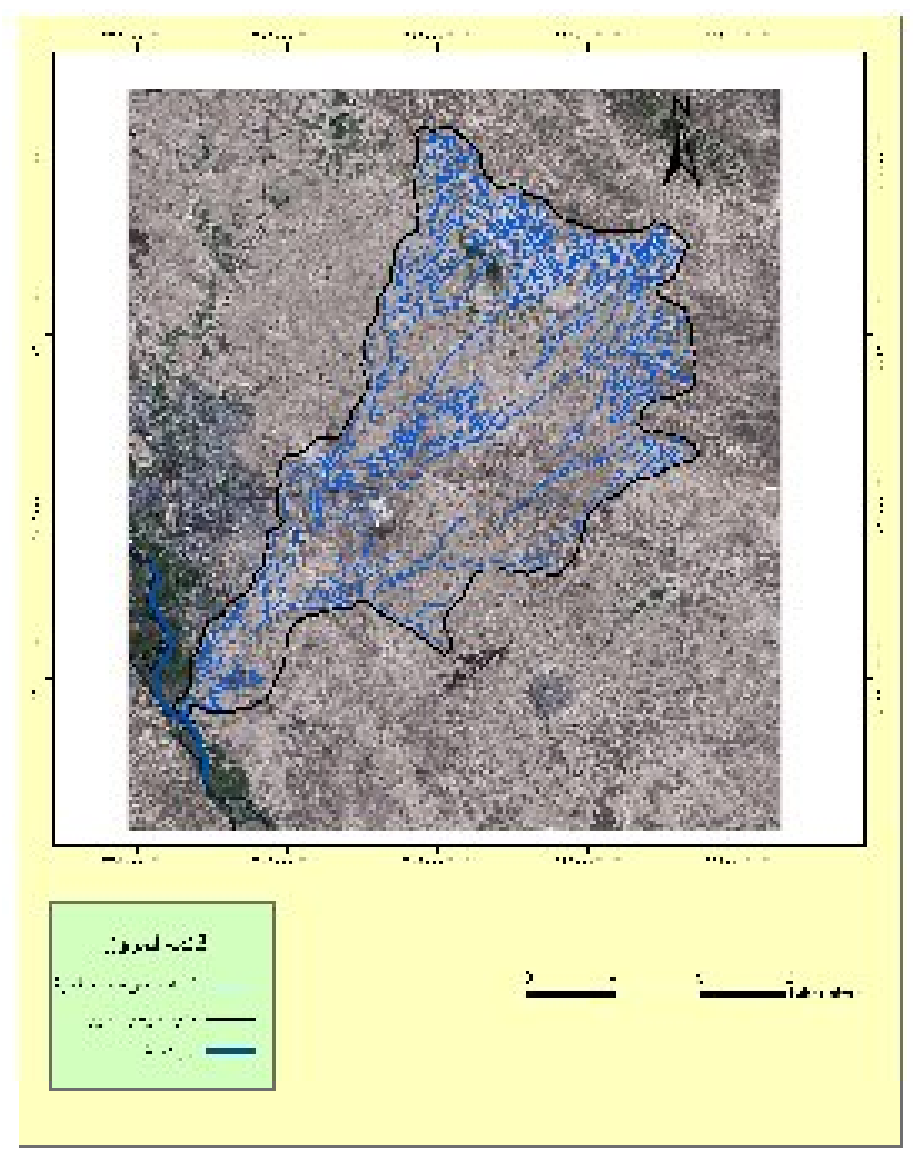

الشكل r: شبكة التصريف السطحية منتجة باستخدام برنامج (Arc map v.9.0). 
وبالاعتماد على معدل الساقط المطري والذي يبلغ (YV ملح) لمنطقة الدراسة والذي تم الحصول عليه

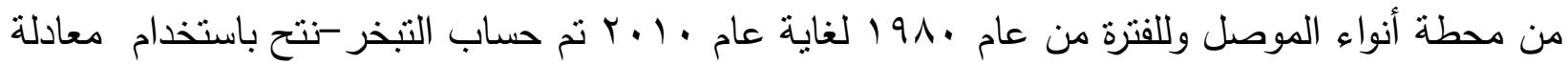

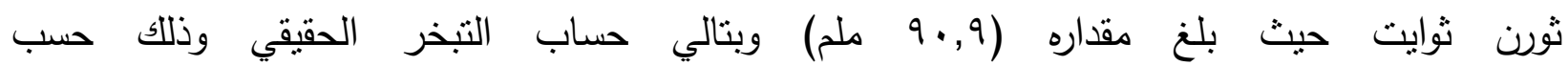

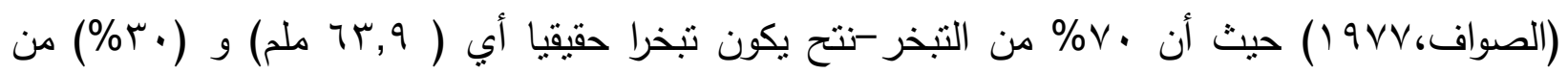
التبخر -نتح يكون نتحا أب (VV ملم).تم الاعتماد على معدل الارتثاح لمنطقة الدراسة في حساب الجريان

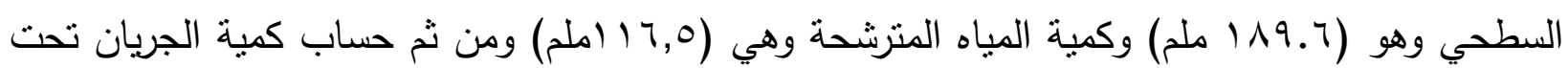

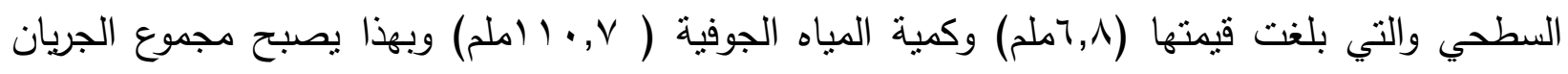

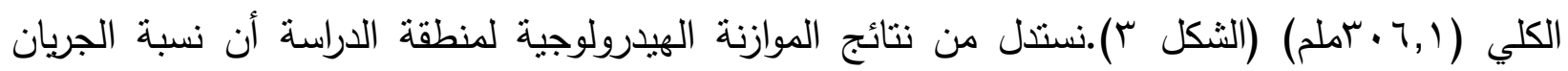

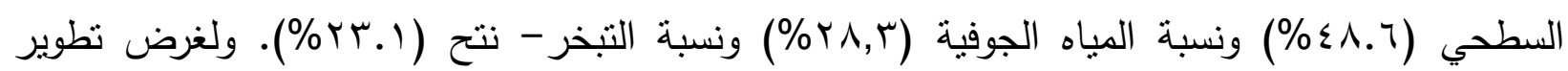
الآبار الموجودة في منطقة الدراسة يجب زيادة نسبة المياه الجوفية التي تعتبر منوسطة على حساب نسبة التباه الجريان السطحي.تم رصد واحد عشرون بئرا ارتوازيا في منطقة الدراسة وقد تم التركيز على لثلى ثمانية أبار

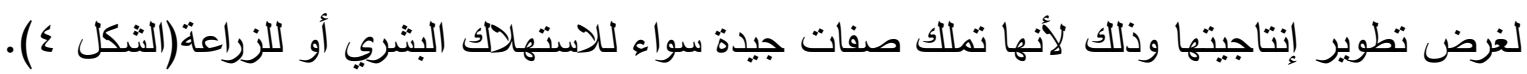

\section{ثالثا": التحليل المورفومتري لحوض وادي الثور.}

جرت العادة في إجراء التحليل المورفومتري لأي شبكة تصريف سطحية القيام بالقياسات التي تتعلق

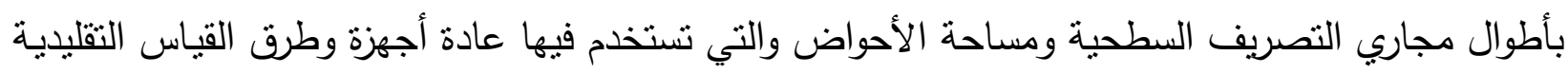

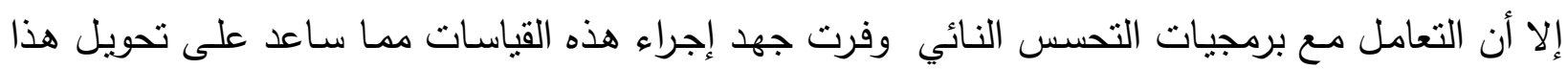
التحليل من طريقة إجراءه الروتينية إلى استخدام الحاسوب وإعداد البيانات الجدولية الداخلة في هذا التحليل.
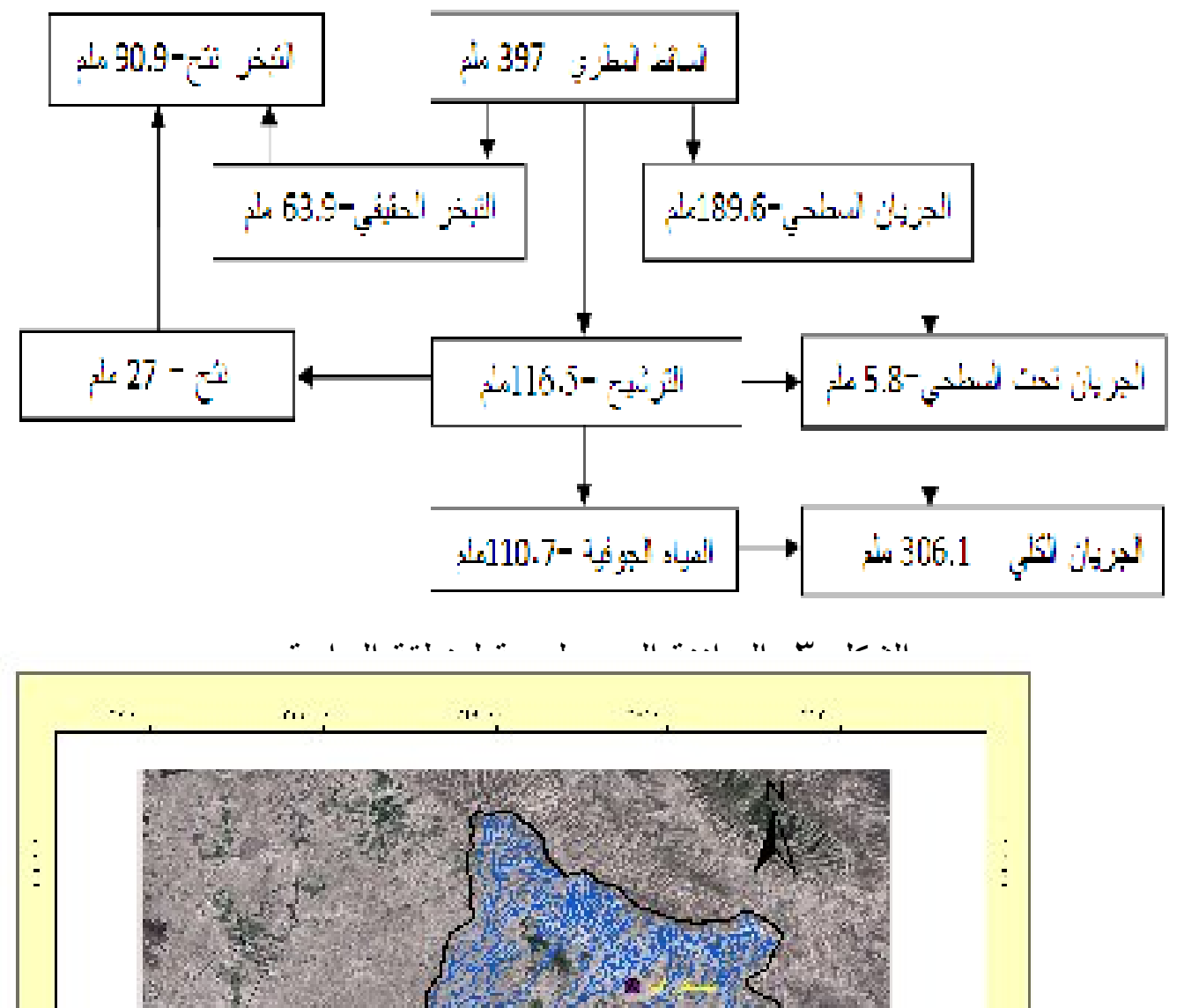
لغرض وصف هندية حوض التصريف ونظامه النهري بصورة جيدة نحتاج إلى قياسات عديدة لعناصر شبكة التصريف السطحية ومساحة الحوض والارتفاع الطوبوغرافي وانحدار الثبكة. تم استخدام برنامج (Arc view v.3.3) في إجراء التحليل المورفومنري وإيجاد المتغيرات المورفومنرية لحوض وادي

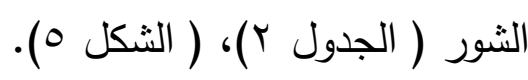
مناقثة نتائج التحليل المورفومتري

تشير نسبة تماسك المسـاحة إلى مدى تقارب أو تباعد شكل الحوض عن الشكل الدائري وتتحصر قيمتها بين الصفر والواحد فالقيم المرتفعة والقريبة من الواحد تعني عادة وجود أحواض مستديرة الثكل وهذا ما ظهر في نتيجة حوض الثور حيث بلغت (rT. · ) ويمكن تقسير هذه النتيجة على تقدم الحوض في دورته الحتية. تعد أهمية هذه الخاصية في معرفة مدى سرعة وصول موجات الفيضان إلى المجرى الرئيسي 
وتتحصر قيمتها بين الصفر والواحد ، وقد ظهرت نتيجتها (V . .) أي قريبة من الصفر وتدل هذه القيمة إلى

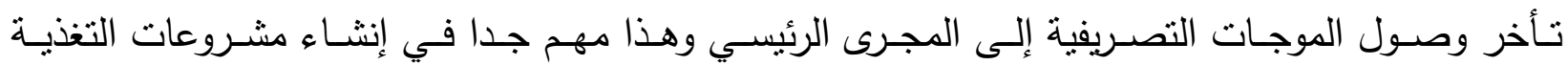
الاصطناعية وذلك لضمان خزن اكبر كمية من المياه الموسمية في الحشارج المائية أسفل حوض الوفئ الوادي.

الجدول r: قيم بعض المتغيرات المورفومترية لحوض وادي الثور المحسوبة باستخدام

برنامج (Arc view v.3.3)

\begin{tabular}{|c|c|c|c|c|}
\hline لأبية & لنسنتز أحبردئمنزي & لنيسة: & 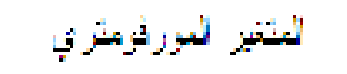 & \\
\hline 108 & 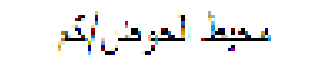 & 303.4 & 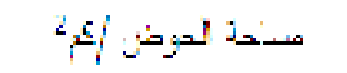 & \\
\hline 39.5 & 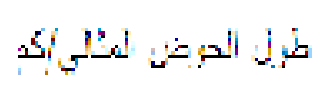 & 47.6 & 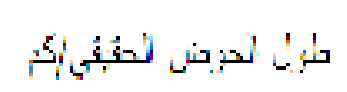 & \\
\hline 0.37 & نيبد "النتثلة: & 0.65 & نبة الإيتـلة & $a^{2} 3$ \\
\hline 0.14 & 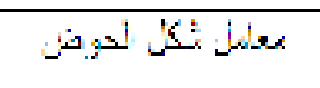 & 0.62 & 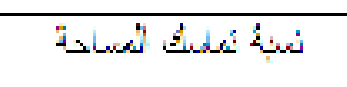 & \\
\hline $5<9$ & ميد أمجزي. & 6 & 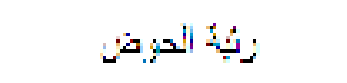 & \\
\hline-.78 & 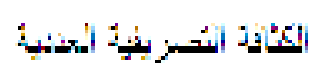 & 3.88 & نبّة المتَّبَ & $\sqrt[3]{3}$ \\
\hline-31 & 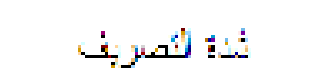 & 2.34 & 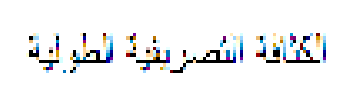 & . \\
\hline
\end{tabular}

تـتحكم خاصـية الاسـتطالة بدرجـة كبيـرة بكميـة الميـاه التـي تجهـز المجـرى الرئيسـي للحـوض

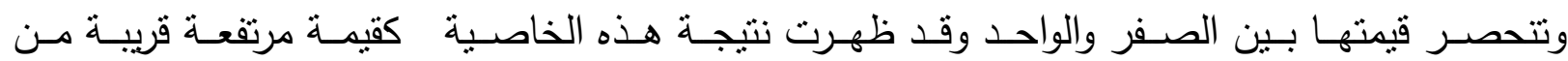
الواحد(10. . ) وتثير إلى أن المجرى الرئيسي لحوض الوادي يستلم كميات كبيرة من مياه السيح الموسمي.

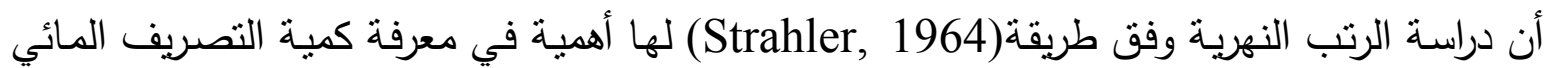
والذي له انعكاس على قدرة هذه الأودية الحتية والإرسابية وبالتالي على الحد من نأثيراتها على استعمالات

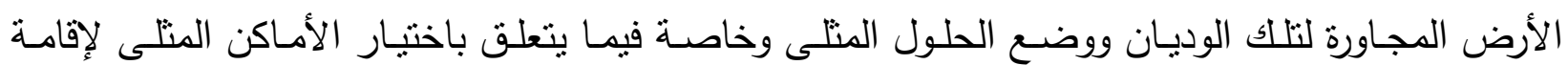
مشروعات التخذيـة الاصطناعية، وقد ظهرت نتائج تحليل المراتب النهرية لحوض وادي الثـور من الرتبـة السادة حيث تتير هذه الرتبة إلى استلام هذا الحوض لكميات كبيرة من مياه الموسمية على شكل جريان

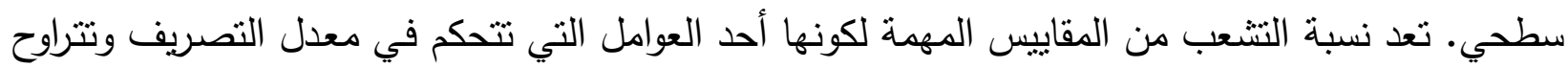


قيمتها بين (r-0) للأحواض الطبيعية وقد اثبت (Mc Cullaph, 1986) إن هناك علاقة مابين طول فترة

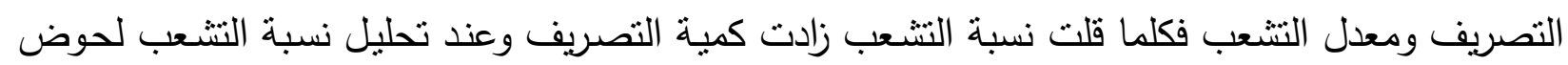

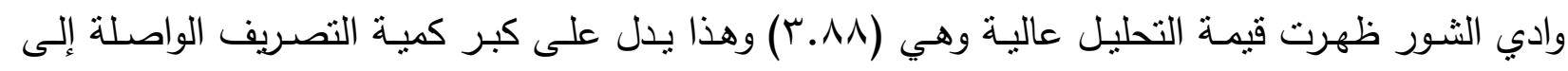

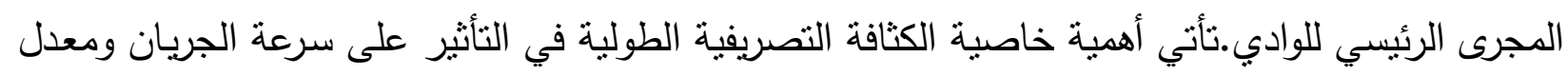

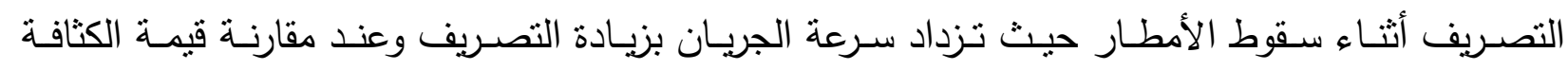

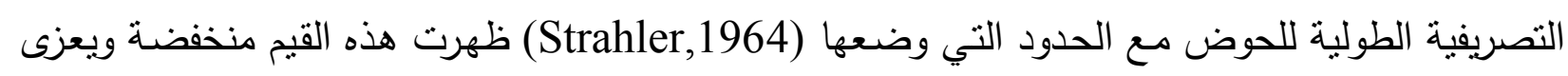
ذللك إلى أن معظم أجزاء هذا الحوض واقعة في مساحات ذات الحدار قليل مما يسمح بنفاذ كميات كبيرة من

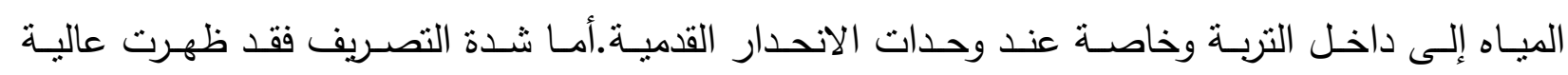
نسبيا( آ. (1) مما يدل على كبر كميات المياه الموسمية المستلمة.

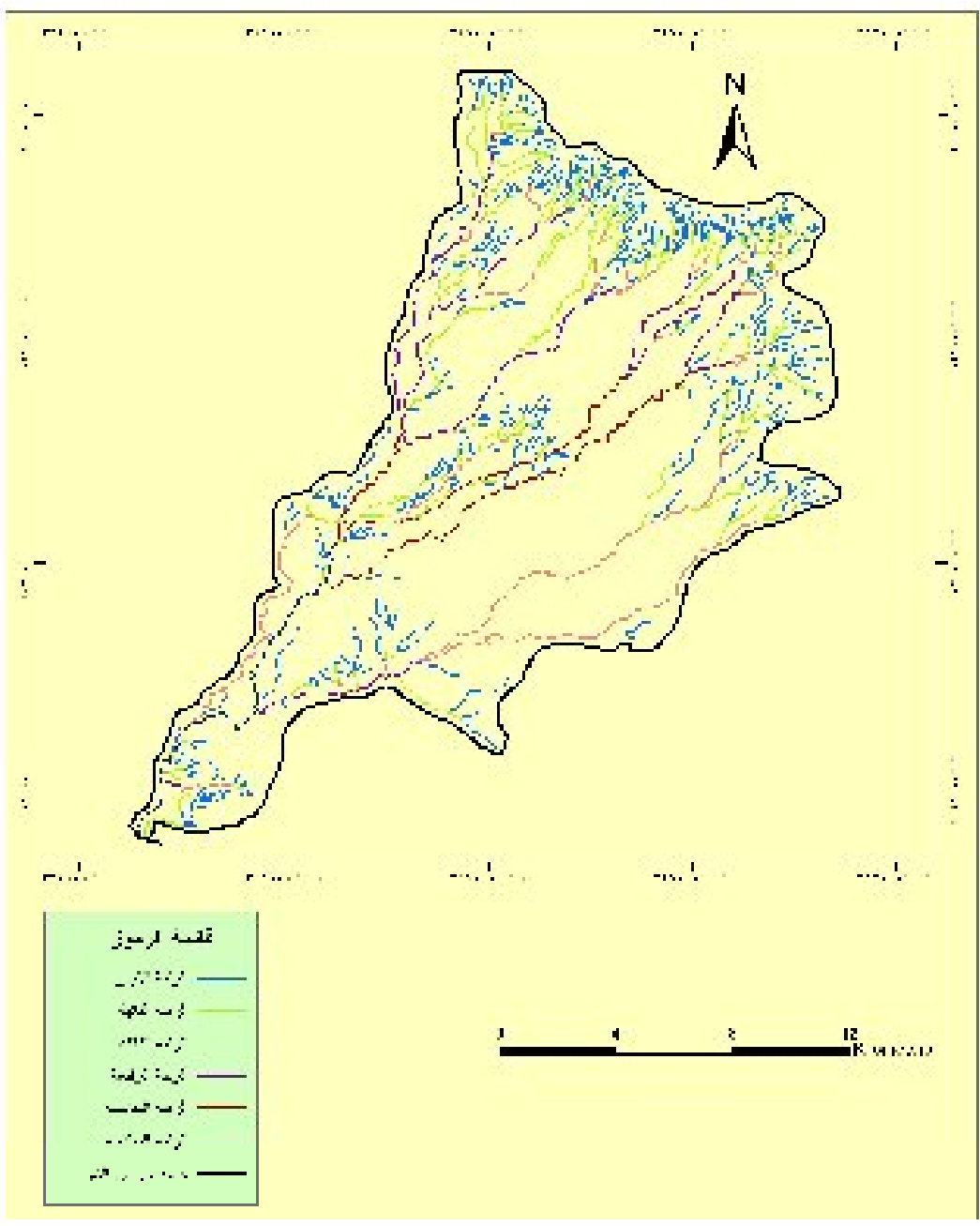

رابعا": تحديا طريفه حصاد مياه الامطار وتصميم الحواجز الحجريه ألحزيه

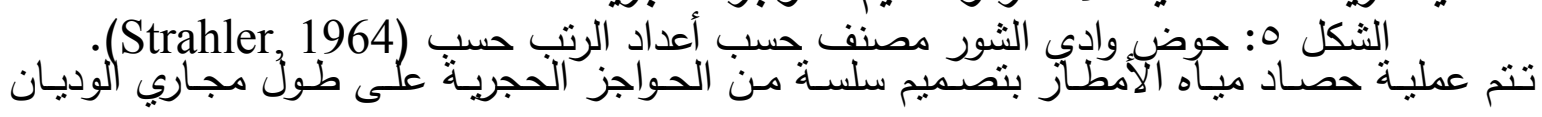

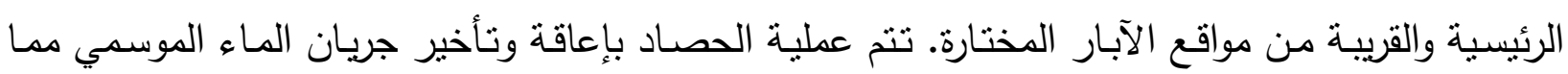


يطيل ذلك زمن مغاض المياه داخل قطاع التزبة ومن ثم ترشحه إلى التكوينات غير المحصورة في منطقة الدراسة( الثكل جا ).

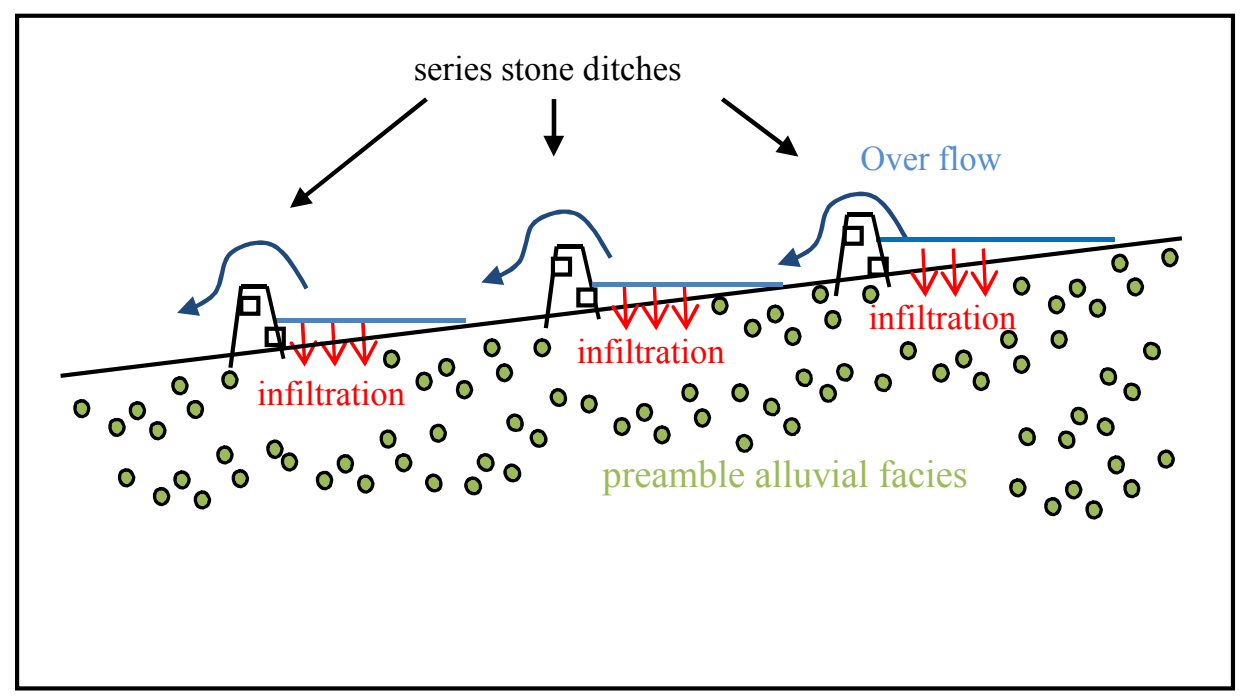

الثكل 7: حصاد مياه الأمطار باستخدام الحواجز الحجرية.

يتكون جسم الحاجز من حجارة مختلفة الأحجام مع طبقة كونكريتية عمودية في (upstream) الغاية منها عدم نفاذ المياه المتجمعة خلف الحاجز إلى جسم الحاجز مما يؤدي إلى حدوث التآكل والنحر مع مرور الزمن وبكون سمك هذه الطبقة (• (1) سم. أما في (downstream) فيكون شكل الحاجز مدرج والغاية من هذا التصميم هو تخفيف طاقة مياه السيح السطحي في الحالات التي يحدث فيها (over flow) وتكون هذه الدرجات مغطاة بطبقة كونكريتية بسمك (• (1) سم (الشكل م) (Travers et.al.,1991). تم اختيار أفضل المواقع لإقامة الحواجز الحجرية وعلى طول المجاري الرئيسية للوديان وبالقرب من موقع الآبار وذلك لضمان حدوث عملية الحصاد بصورة جيدة ونفاذ اكبر كمية من مياه السيح السطحي لتغذية وزيادة الإنتاجية السنوية تم استخدام برنـامج(Global mapper v.11) في إيجاد المقطع العرضي للحواجز الحجريـة وحساب.

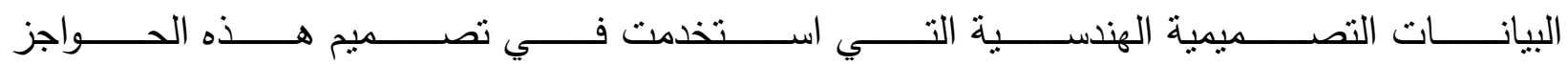
( الثكل ^). 
خامسا": إعداد الخارطة الاكترونية

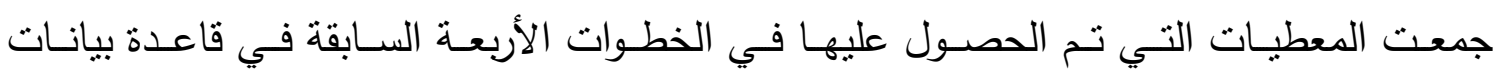

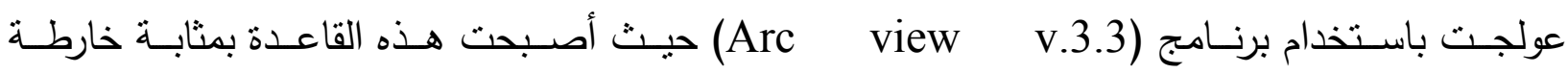

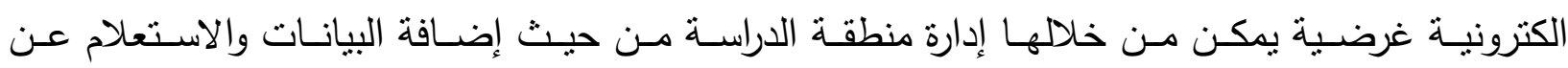

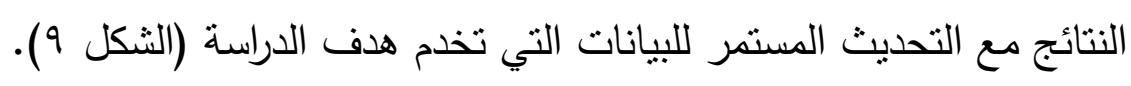

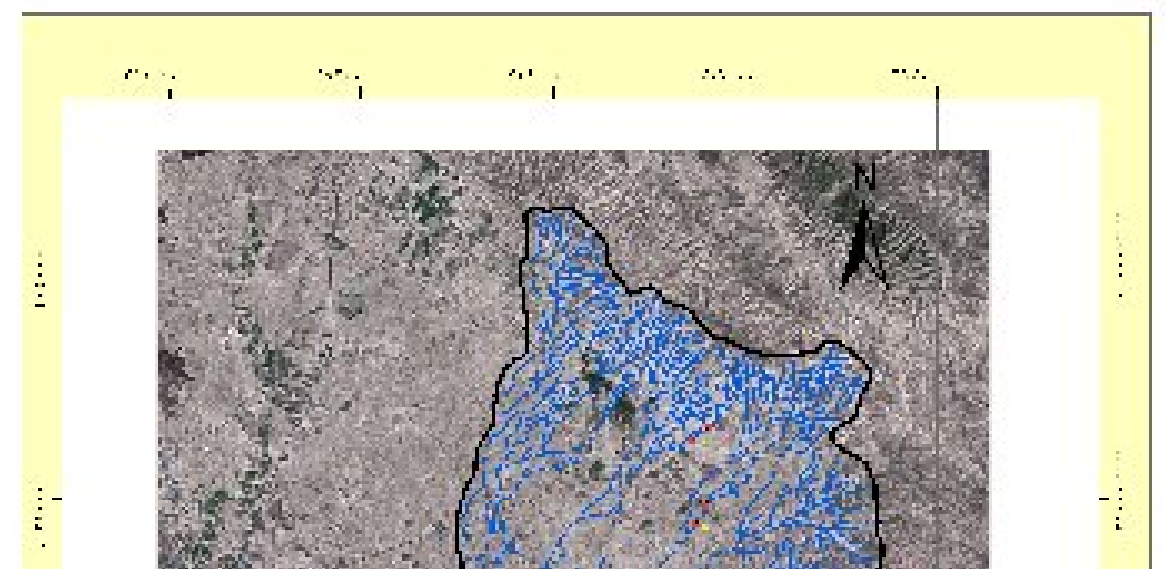




\section{النتائج والمناقشة}

تم اعداد قاعدة معلومات لمنطقة الدراسة مكونة من تحليل مورفومتري وتحليل هيدرواوجي بالاعتماد على معطيات التحسس النائي والبيانات المناخية والدراسات السابقة لمنطقة الدراسة واعداد خرائط غرضية متمثلة بشبكة التصريف السطحية (الشكل r) وخارطة التحليل المورفومتري لمنطقة الدراسة (الثكل ه). حيث اسفر تحليل الموازنة الهيدرولوجية (الثكل r) تحديد كميات المياه الداخلة والخارجة من الحوض مع تحديد كمية المياه الجوفية التي تخزن سنويا، كما اسفر التحليل المورفومتري للحوض على ملائمة صفات وخصائص الحوض المورفومترية (الجدول r) على ثتفيذ مبدا التغذية الاصطناعية لمياه السيح الموسمي عن طريق تطبيق مبدا حصاد مياه الامطار والذي تمنل ذلك باختيار طريقة انشاء الحواجز الحجرية القاطعة لمجاري الوديان الرئيسية.تم الاستعانة بالدراسات السابقة في رصد وتحديد الابار الموجودة في منطقة الدراسة حيث تم ادخال البيانات التي تتعلق بالابار من حيث انواعها وخصائصها الكيميائية التي تضمنتها هذه 
الدراسة في قاعدة المعلومات حيث تم رصد واحد وعشرين بئر تم اختيار ثمانية ابار منها وذلك لملائمة صفاتها الكيميائية مع المواصفات العالمية من حيث كون مائها صالح للاستهلاك البشري وللزراعة والانتهاء باعداد خارطة غرضية تمنل توزيع هذه الابار (الثكل ع).

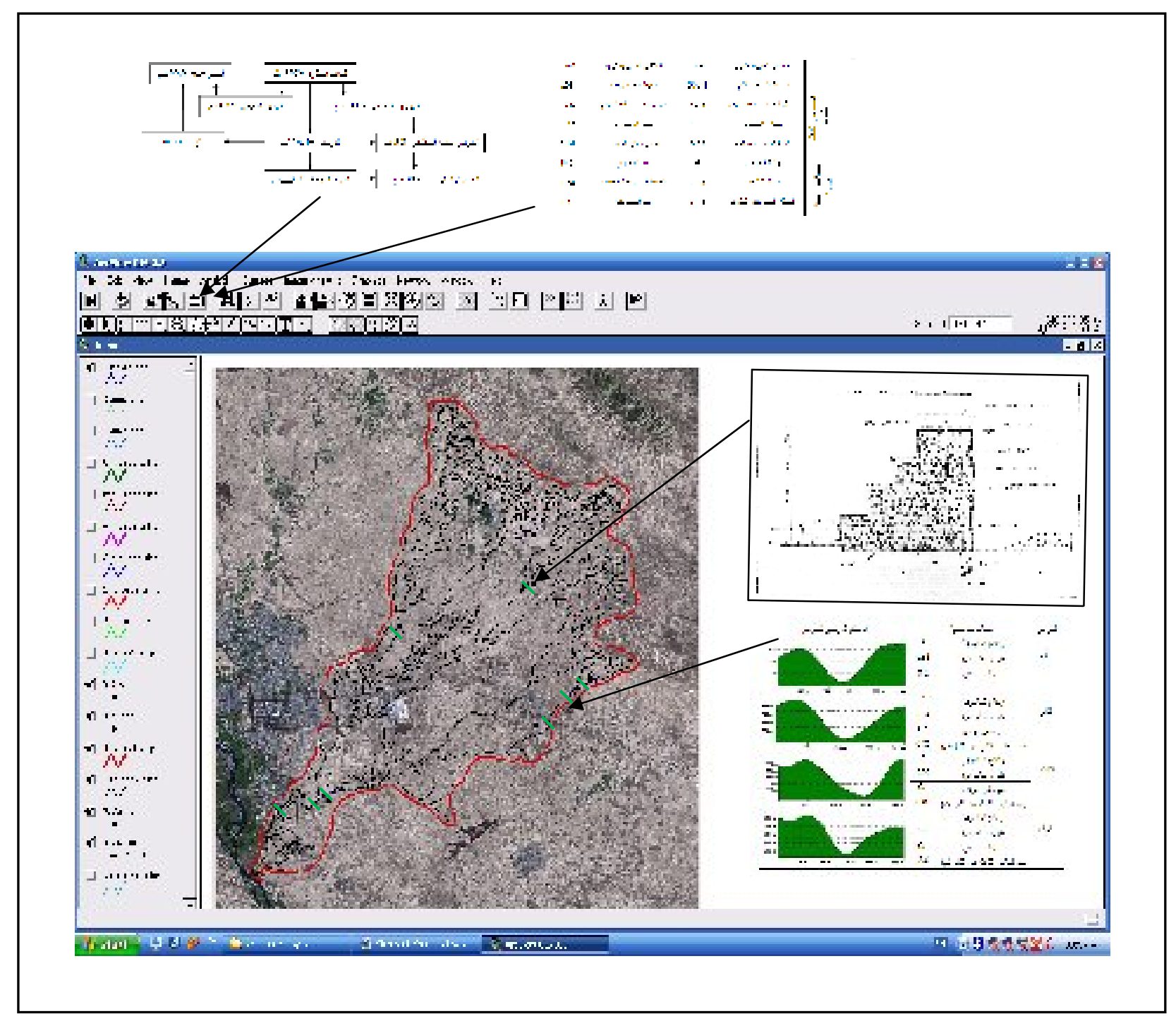

الثكل 9: الخارطة الإكترونية لمنطقة الدراسة والمنتجة ببرنامج (Arc view v.3.3).

تم جمع مستويات البيانات التي تم تحليلها اضافة الى بيانات الدراسات السابقة واعداد بنك للمعلومات

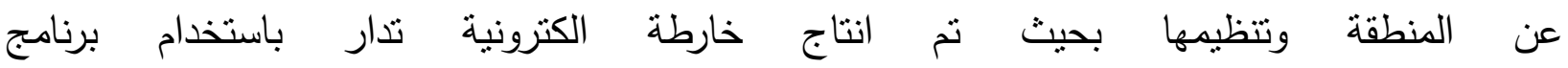
(الشكل 9 ) حيث ان الفائدة من هذه الخارطة هي المتابعة المستمرة لمشاريع التغدية 
الاصطناعية في المنطقة حيث تعطي هذه الخارطة مرونة في ادارة البيانات المنوفرة عن المنطقة مع امكانية تحديثها سنويا وايجاد الحلول المناسبة لصيانة هذه المشاريع.

\section{الاستنتاجات}

اوضحت الدراسة اهمية استخدام برمجيات نظم المعلوات الجغرافية في ادارة وتحليل البيانات الداخلة في تصميم مشاريع حصاد مياه الامطار واعداد الخرائط الكتورنية التي تستخدم قي تصميم وادارة هذه المشاريع مع مرونة التحديث المستمر والاستعلام عن البيانات الداخلة في تصميم هذه المشاريع التي يمكن ان تعد من المشاريع الاستراتيجية لما لها من اهمية في تطوير منطقة الدراسة من الناحية الزراعية والاستهلاك البشري للمياه الصالحة للشرب من خلال تطوير الابار في منطقة الدراسة.

\section{المصادر العربية}

الجبوري، مرعي ياسين حمود. مورفومتريـة حوض الثـور دراسـة مقارنـة لنتائج استخدام مصـادر البيانـات المتعددة وتقاناتها، اطروحة دكتوراه غير منشورة، كلية التربية، جامعة الموصل، 9 ـ . ؟. النعيمي، فيصل حمادي علي. هيدرولوجية حوض وادي الشور ، رسالة ماجستير غير منشورةكلية العلوم، جامعة الموصل، · ... - ج.

جبرائيل، نذير • تقدير مصادر المياه الجوفية في العراق،معهد بحوث الموارد الطبيعية،مؤسسة البحث العلمي/

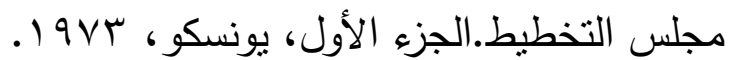

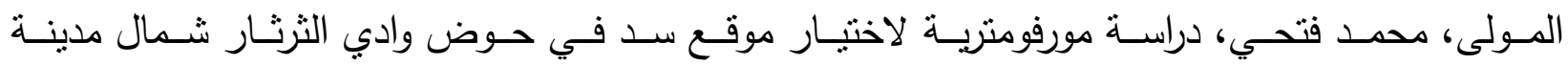
الحضر باستخدام تقنيات التحس النائي، رسالة ماجستير غير منشورة،كلية العلوم، جامعة الموصل، $. r . r$ وزارة النقل والمواصلات، ـ ـ ـ.الهيئة العامة للأنواء الجوية،شعبة المناخ، جداول غير منشورة. المصادر الأجنبية

WHO, 1971. International Standards for Drinking Water World Health Organization, 3rd-ed, Genava, Switzerland, p. 36. 


$$
\text { بسمان يونس حميد و بشار منير يحيى }
$$

Mohamed, B. and Zhank, X., 2008. GIS and Remote Sensing Applications for Rain Water Harvesting in the Syrian Desert (AL-BADIA).Twelfth Internationals Water Technology Conference, IWTC 2008, Alexandria, Egypt. pp. $73-82$.

Kumar, M., 2008. Digital Image Processing, Photogrammetry and Remote Sensing Division. Indian Institute of Remote Sensing, Dehra Dun, pp. 81 - 108, Available at:www.wamis.org/agm/,Accessed at: May-2010.

Strahler, A. N., 1964. Quantitative Geomorphology of Drainage Basins and Channel Network: In a Book of Applied Hhydrology, Edited by Chow, V.T., Mc Graw-Hill, New York.

Mc Cullaph, P., 1986. Modern Concept in Geomorphology, Oxford Univ. Press. Oxford, England.

Travers M., Rofr K and Lapowerth H., 1991. Hydraulic Structure in Jabal Al-akhdar, Inception Report Primary Design for the Ministry of Agriculture and Fisheries of the Sultanate of Oman. 